\title{
RE-PENSANDO A LA PLANIFICACIÓN COMO MÉTODO DE TRABAJO
}

\section{Rethinking Planning as a Working Method}

Fecha recepción: 27 de noviembre de 2021 / fecha aceptación: 14 de diciembre de 2021

\section{Arlette Pichardo Muñiz}

\section{Cómo citar este artículo:}

Pichardo Muñiz, A. (2021). Re-pensando a la planificación como método de trabajo. Revista Pensamiento y Acción Interdisciplinaria, 7(2), 114-127. https://doi.org/10.29035/pai.7.2.114

\section{Resumen}

Re-pensando a la Planificación como método de trabajo forma parte de un esfuerzo teórico-metodológico y práctico más amplio, realizado y publicado por la autora bajo el título "De cómo hacer Planificación Situacional aprendiendo" (Pichardo, 2021). En esta oportunidad, a partir de la categoría situación se describe-de manera sucinta-la fundamentación epistémica de la propuesta, base sobre la cual se edifica el mapa situacional. Al mismo tiempo, se presenta un conjunto de consideraciones fundamentales de cara a una resignificación de la planificación como método de trabajo. Dicha propuesta se focaliza en incorporar, desde una acción mancomunada, funciones esenciales relativas a la promoción, a la indagación, a la programación, a la organización (incluyendo a la coordinación y a la administración) y a la evaluación. De ahí la explicación del nacimiento y sustento del Modelo PipoE, identificado por las siglas de tales componentes integrales o dimensiones consustanciales. La intencionalidad básica, producto de la recapitulación y sistematización de más de 25 años de experiencia en un amplio espectro de organizaciones, es contribuir a trascender las visiones convencionales de la conceptualización y la práctica de la planificación más usuales y frecuentes en el imaginario institucional y social.

Palabras clave: administración, coordinación, evaluación, indagación, organización, planificación, programación, promoción

\footnotetext{
1 Nacida en Santo Domingo, República Dominicana. Es doctora en Educación, con énfasis en Mediación Pedagógica de la Universidad de La Salle en Costa Rica. Cursó la Maestría en Planificación de Desarrollo, especialidad en Planificación Social, en el Centro de Estudios del Desarrollo (CENDES) de la Universidad Central de Venezuela. Licenciada magna cum laude en Sociología en su ciudad natal. Autora de "Planificación y Programación Social", "Evaluación del Impacto Social", "Ábreme la puerta. Casa de Aprendiencia de la Política Social y otras políticas", entre otras publicaciones de libros con sello editorial, artículos de difusión científica en revistas especializadas, informes de evaluación y documentos técnicos. Catedrática (retirada) de la Universidad Nacional (UNA) en Costa Rica. Reconocida como Mujeres Pioneras de esa universidad por ser primera directora general del Centro Internacional de Política Económica para el Desarrollo Sostenible (CINPE). Actualmente, se desempeña como Consejera de Evaluación de gobiernos y entidades no gubernamentales. Correo electrónico: arlettepichardo@gmail.com
} 


\begin{abstract}
Rethinking Planning as a Working Method is a integral part of a broader theoretical methodological and practical effort, carried out and published by the author with the title (in Spanish) "De cómo hacer Planificación Situacional aprendiendo" (Pichardo, 2021). On this occasion, based on the situation category, it describes -succinctly- the epistemic foundation of the proposal, the basis on which the situational map is built. At the same time, it presents a view about redefinition of planning as a working method. The proposal has focus on essential functions related to the promotion, the research, the programming, the organization (including the coordination and the management) and the evaluation. Hence the explanation of the birth and sustenance of the PipoE Model (by acronym in Spanish) identified by the initials of its integral components and consubstantial dimensions. The basic intention, product of the recapitulation and systematization of more than 25 years of experience in a wide spectrum of organizations, is to contribute to transcending the conventional views of the conceptualization and practice of planning that are more usual and frequent in the framework institutional and the social imaginary.
\end{abstract}

Keywords: coordination, evaluation, organization, management, planning, programming, promotion, research

\title{
Introducción
}

El presente artículo contiene un extracto de la disertación de la autora el 10 de noviembre de 2021, como parte de las actividades commemorativas del Día Internacional del Trabajo Social, organizadas por las Escuelas de Trabajo Social de Curicó y de Talca, de la Facultad de Ciencias Sociales y Económicas de la Universidad del Maule, en Chile. En este marco, la exposición se realiza en el espíritu de incitar y animar a una reflexión participativa y constructiva acerca de los posibles usos, desde la práctica del Trabajo Social, del planteamiento central de re-pensando a la planificación como método de trabajo.

En primer lugar, se expone brevemente el marco referencial, desde el punto de vista del contexto histórico en que se origina y sustenta la intencionalidad primaria de la propuesta de re-pensando a la planificación como método de trabajo. En segunda instancia, se describe a modo de síntesis la conceptualización de la categoría situación, núcleo central del marco referencial de dicha propuesta, que se concatena en la edificación del mapa situacional. El tercer apartado corresponde a una descripción de las bases fundamentales del Modelo PipoE, visto a través de sus funciones esenciales, esto es: Promoción, indagación, programación, organización y Evaluación, de donde se deriva su forma de denominación; así como una delimitación del principal papel que le asisten como parte del conjunto que forman parte y un breve relato de sus principales instrumentales de apoyo y asuntos claves a considerar. Para concluir planteando la necesaria interacción dinámica entre estos componentes integrales o dimensiones consustanciales, en la mira de trascender las visiones convencionales más socorridas de la conceptualización y la práctica de la planificación en diversos ámbitos del acontecer institucional y organizativo. 


\section{Marco referencial}

En el último decalustro hemos asistido a una amplificación de los esfuerzos por y para re-pensar a las visiones convencionales de los objetos de estudio, la planificación incluida. Desde nuestra perspectiva, en el caso de esta última tales esfuerzos tendrían que orientarse a reconciliarla con sus orígenes iniciales, en tanto método de trabajo, que precede y preside a la acción. Dos palabras claves que aprendimos de nuestro maestro Carlos Matus, el genio de la fantasía creadora en materia de Planificación Estratégica Situacional (1981, p. 29; cursivas nuestras).

Más allá de la herencia subsistente de formas anteriores de concebir y practicar a la planificación, su dicotomía o antagonismo con el mercado, en tanto mecanismo por excelencia para la asignación de recursos, ha tendido a superarse. De forma similar, el sesgo ideológico derivado de las experiencias históricas de planificación societaria, provenientes tanto de los ex países socialistas, en tanto sustitituvo del mercado, como del capitalismo avanzado, entendido como técnica correctiva de las fallas del mercado. El caso de la URSS, con el Plan de Electrificación Rural o de Francia y la iniciativa del Plan Marshall que, en su momento, constituyeron íconos a replicar y/o evitar.

En términos de balance, podríamos decir que -a grandes rasgos- la planificación es hoy comúnmente aceptada en las prácticas institucionales de entidades públicas, como de empresas privadas y organizaciones sociales diversas. En fin, en todo tipo de ente organizativo, con independencia de su forma de estructuración y conformación, más allá de las visiones estructurales o versiones en boga y las limitaciones inherentes a las conceptualizaciones adoptadas y deficiencias en su aplicación.

Avances notables se han venido realizando en la incorporación de espacios y técnicas de participación en los esfuerzos de planificación, en particular desde experiencias multiactores, en el plano nacional y corporativo, incluso desde el territorio. La tendencia notable es a superar las concepciones verticales inicialmente asociadas de manera exclusiva con el ideario de líderes políticos, o bien las directrices emanadas desde cuadros gerenciales y directivos de nivel técnico. No obstante, hace falta avanzar en procura de un alcance más amplio y un impacto más significativo.

Este panorama, brevemente expuesto, sin lugar a dudas abre un espacio de oportunidad para el desarrollo de aproximaciones diferentes y sustentables, en busca de superar limitaciones inherentes a las visiones convencionales de las conceptualizaciones y prácticas de la planificación en el entorno institucional y en el imaginario social. Es a partir de estas consideraciones básicas que se edifica la propuesta de re-pensando a la planificación como método de trabajo. 


\section{La categoría situación y el mapa situacional}

En la base de la propuesta de re-pensando a la planificación como método de trabajo, internalizamos a la situación en tanto categoría epistémica. En términos simples, se trata de un concepto común, de fácil entendimiento y sencillo en su comprensión.

Su utilización y aportes primigenios en la planificación estratégica corresponden a Matus (1977). Este autor indica que inicialmente acude a esta categoría de manera intuitiva y, con el paso del tiempo, la va referenciando a partir de connotados pensadores. Al respecto, quizás como uno de los principales referentes de su obra, plantea la siguiente definición:

Situación es donde está situado algo; ese algo es el actor y la acción. Acción y situación conforman un sistema complejo con el actor. La realidad adquiere el carácter de situación en relación al actor y a la acción de éste. Por eso, una misma realidad es al mismo tiempo muchas situaciones, dependiendo ello de cómo está situado el actor y cuál es su acción...De manera que la situación es todo aquello que es relevante para la acción...La situación es condicionante del actor y de la acción, y la acción es eficaz en la construcción de la situación. (1977, p. 70)

Desde la categoría situación es que asumimos la edificación del mapa situacional. El propósito es la búsqueda de una aproximación dinámica con mejores posibilidades de identificar y utilizar espacios estratégicos para la acción que los esquemas lineales o incluso matriciales. El concepto situacional lo incorporamos en tanto sustantivo propio y no como un simple adjetivo calificativo. Se trata de un nombre con resonancia, atractivo y sugerente, para imprimir sentido esencial y significación sustantiva, al marcar en forma disruptiva una diferenciación conceptual y teórico-práctica, trascendiendo a la simple utilización de un apelativo, más allá de la semántica. 


\section{Figura 1}

Mapa Situacional

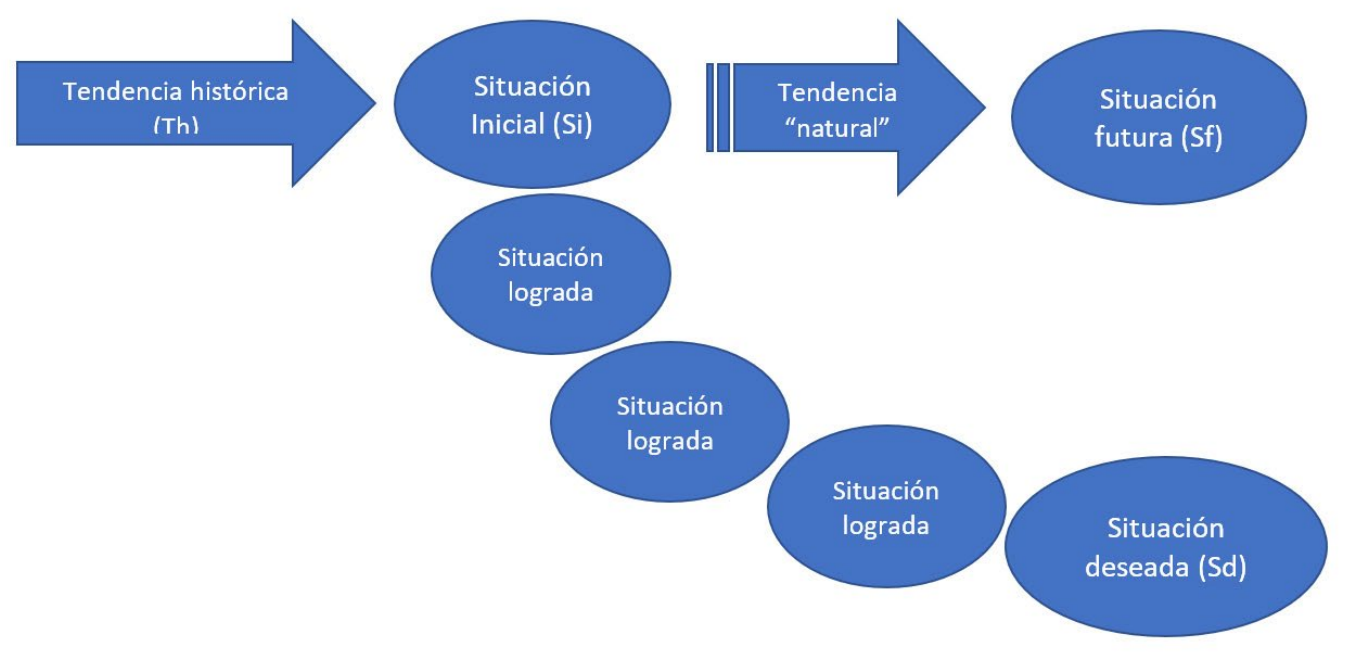

Fuente: resumido de Pichardo, 2021, p. 66.

En el contexto de la edificación del mapa situacional es posible desglosar multiplicidad y variedad de situaciones. Desde el punto del vista del horizonte temporal, en principio, podemos diferenciar y distinguir una tipología de, al menos, cuatro tipos de situaciones: situación inicial (Si), situación futura (Sf), situación deseada (Sd) y situación lograda (SI).

i. Situación inicial. Es la situación de donde parte el esfuerzo de re-pensando a la planificación como método de trabajo. Su intención fundamental es identificar, describir y caracterizar al problema focal, objeto central de la acción planificada, con el fin de ubicar y posicionar espacios estratégicos para actuar, a partir de su conceptualización y contextualización en función de la tendencia histórica que lo origina y explica.

ii. Situación futura. Es la situación, por así decirlo, a que se llegará de manera inevitable ante la ausencia, por así decirlo, de re-pensando a la planificación como método de trabajo. Por tanto, es la situación que se quiere evitar u obviar, o bien a la que no se quiere llegar, pero que se llegará de manera inevitable por evolución "natural" (para llamarle de alguna manera) de la situación inicial en función de la tendencia histórica, a menos que la acción planificada tenga capacidad para cambiar ese rumbo y orientar el proceso de planificación hacia la situación deseada. 
iii. Situación deseada. Es la situación de mayor interés para los propósitos de re-pensando a la planificación como método de trabajo. Se trata de la esencia, la intencionalidad o el leit motiv, pues el propósito fundamental de la acción planificada es cambiar el curso de los acontecimientos de la tendencia "natural" para, en su lugar, enrumbarse hacia una Sd, sustancialmente distinta y diferenciada de la Sf, que implique la atención del problema focal, objeto central de la planificación, identificado, descrito y caracterizado en la $\mathrm{Si}$, en tanto producto de acumulaciones esenciales derivadas de la tendencia histórica.

iv. Situación lograda. Es la situación, o más bien el conjunto de situaciones, que desde re-pensando a la planificación como método de trabajo se van alcanzando en forma intermedia en el tránsito desde la Si hacia la Sd.

Resulta útil recalcar que una pregunta inicial que engarza al mapa situacional, aunque puede plantearse de mútiples maneras, en su aproximación primaria implica plantear:

¿De qué manera se combinan las condiciones de la situación inicial para obviar o evitar la situación futura y encaminarse hacia la situación deseada y su expresión en situaciones logradas?

En ese sentido, también es útil recordar que pequeñas diferencias en un sistema dinámico pueden desencadenar enormes y, a menudo, insospechados resultados. El conocido efecto mariposa. Para algunas personas alude a una exageración y, efectivamente, así es, pero pone sobre la mesa la necesidad de indagar sobre el poder de la influencia sutil.

Así como que una misma situación admite pluralidad de relatos, en función de cómo se sitúen los actores. Al respecto, a modo de ilustración, cabe recordar una conocida anécdota que se le atribuye a Bernard Shaw (1856-1950):

Con una herramienta arreglaba el antejardín de su casa, la vecina que iba de compras le dice en tono de pregunta afirmativa: ¿Trabajando Sr. Shaw? y él responde: "no vecina, estoy descansando".

Al regreso de las compras, la vecina observa que Shaw está ahora en su silla fumando su pipa con la mirada perdida en el horizonte. Entonces, ella le dice: ¿Descansando Sr. Shaw? y él responde distraído: "no señora, trabajando". 


\section{Modelo PipoE}

El Modelo PipoE, como ya indicado, es una creación y aplicación por más de 25 (veinticinco) años, desde diferentes espacios de actuación -universidades, organismos internacionales, instituciones públicas, empresas privadas, cooperativas y organizaciones sociales de la más amplia diversidad- y ámbitos diversos de toma de decisiones - directivo, gerencial, operativo, de base y núcleos primarios-.

La noción de modelo la utilizamos en su sentido amplio, arquetipo o punto de referencia, que implica una representación simplificada. Su nombre procede de las siglas de sus funciones esenciales. Vale decir, P en mayúscula, de Promoción; i de indagación; $p$ de nuevo y en minúscula, de programación; o de organización y E en mayúscula, de Evaluación. Para su explicación recurrimos a una metáfora. Imaginemos una sencilla rueda de timón o de comando direccional de una embarcación.

\section{Figura 2}

Modelo PipoE

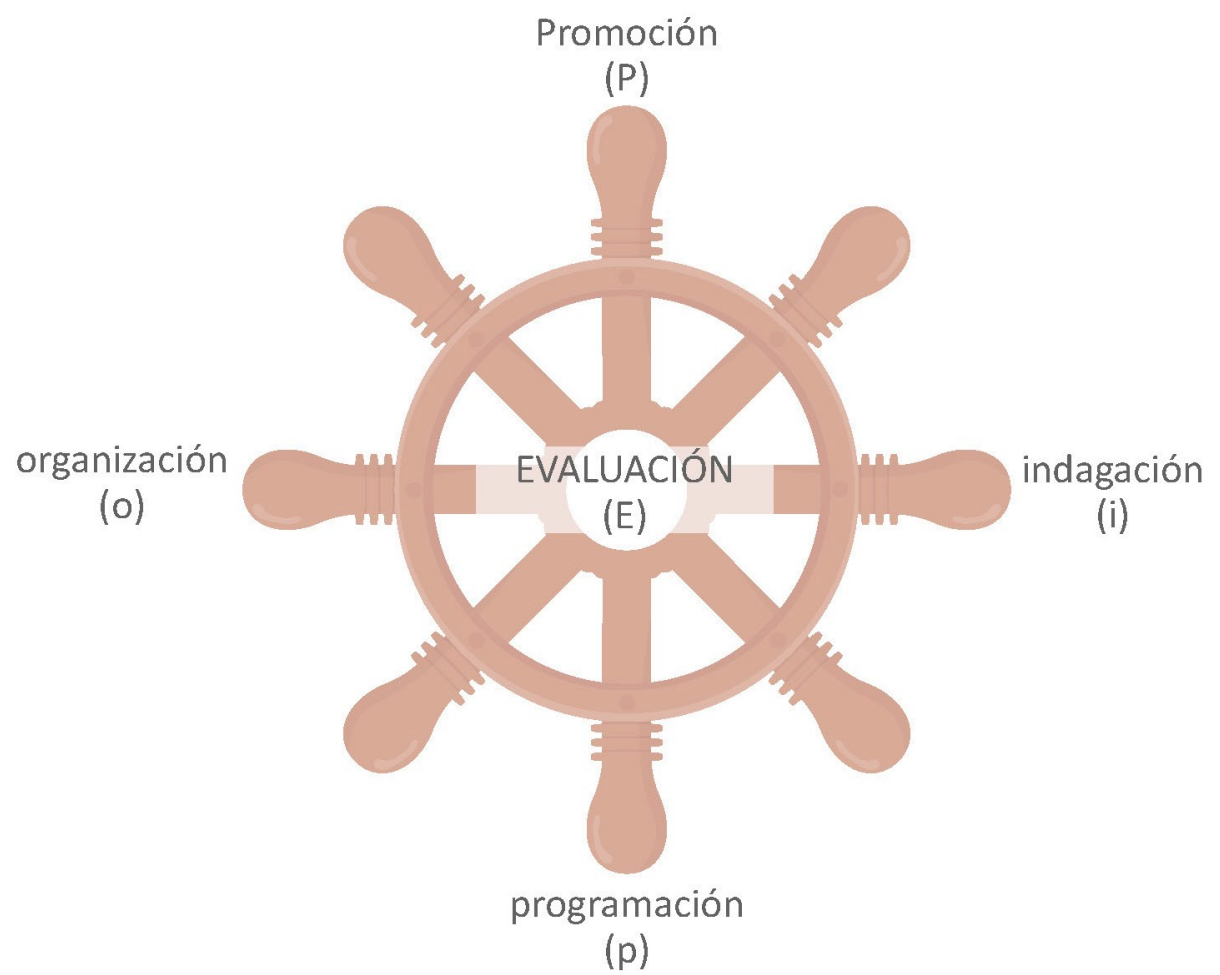

Fuente: Pichardo, 2021, p. 23. 
Desde el modelo PipoE y sus prácticas de aprendizaje, intentamos realizar un aporte de cara a la búsqueda de opciones ante las visiones más generalizadas de la planificación en el contexto del imaginario institucional, tanto en el sector público como en ámbitos empresariales y organizaciones en general, que la alejan de contenido esencial y significación sustantiva en tanto método de trabajo.

Como explicamos en "De cómo hacer Planificación Situacional aprendiendo" (Pichardo, 2021, pp. 156-157):

Para algunas personas, quienes continúan enroladas en las concepciones convencionales de planificación y aun no se acercan a vertientes de reconceptualización y resignificación, el acto de planificar se concibe como un dictado de designios a voluntad, de inexorable cumplimiento, una especie de "camisa de fuerza". Una imagen muy ruda que traemos a colación en el sentido de su uso más común, una prenda diseñada para inmovilizar, con el fin de mantener bajo control a otras personas.

El ejercicio de planificar se focaliza en una o varias personas, cuya capacidad de control se deriva de una especie de clarividencia para "ver" lo que hay qué hacer. Una especie de bola de vidrio o copa de cristal, que "alumbra" la escritura de un documento al que se le denomina política, plan, programa o proyecto.

Los resultados de un ejercicio de esa naturaleza, así concebido y practicado, generalmente contienen una lista de lineamientos o medidas a ponerse en práctica, sin que medie una valoración efectiva de verificación de necesidades y validación de condiciones de viabilidad.

Una lista de chequeo de cosas por hacer. En el mejor de los casos, con buenos deseos. Un plan-libro, como se le acostumbra a llamar en términos despectivos. La norma es el principio de orientación, por tanto el "deber ser" -como dice Matus (1972)- se coloca por encima del "puede ser".

En esta forma de proceder, el criterio de "éxito" está dado por la certeza en la capacidad de adivinar el futuro. El indicador de "fracaso" es la falta de cumplimiento de las acciones, tal como inicialmente se escribieron en los documentos de trabajo. 
Para otras personas, el acto de planificar se concibe y practica como sinónimo de elaborar un listado o inventario de actividades, cuya "bondad" se valora en función de la exhaustividad. Similar a una especie de "carta de intenciones", con buenos deseos enunciados con antelación; sin que, necesariamente, esté asociada con intencionalidad, direccionalidad y viabilidad.

De modo similar a la versión anterior, una persona o más de una enumeran lo que hay que hacer, prevaleciendo de igual manera la noción de lista de chequeo de cosas por hacer.

El criterio de "éxito" al que comúnmente se acude para valorar el mérito de un ejercicio de esa naturaleza es el nivel de desagregación y detalle logrado al identificar el conjunto de actividades junto a la capacidad de antelación, entendida como el tiempo cronológico que medie con respecto a las posibles fechas de ejecución.

El indicador de "fracaso" es la incapacidad para advertir hasta el más mínimo detalle posible los recursos requeridos para ejecutar las actividades; sin preocupación de que respondan a objetivos definidos, metas claramente formuladas e impactos previsibles previamente identificados sobre los cambios o transformaciones buscados.

Con base en las explicaciones anteriores, es posible contextualizar las vinculaciones del Modelo PipoE con la categoría situación, en tanto categoría epistémica como hemos enunciado antes, y especificar tal vínculo en particular a partir del papel y propósito de las funciones esenciales de Promoción, indagación, programación, organización y Evaluación, en tanto componentes integrales o dimensiones consustanciales. 


\section{Tabla 1}

Descripción de funciones esenciales del Modelo PipoE

\begin{tabular}{|c|c|c|}
\hline Modelo PipoE & Papel & Propósito \\
\hline Promoción & $\begin{array}{l}\text { Visibilizar el compromiso } \\
\text { de actuación } \\
\text { problema-actores-acciones. }\end{array}$ & $\begin{array}{l}\text { Fomentar, impulsar, buscar apoyo de } \\
\text { grupos de interés estratégico, con el fin } \\
\text { de promover alianzas estratégicas y/o } \\
\text { contingentes. }\end{array}$ \\
\hline indagación & $\begin{array}{l}\text { Averiguar en } \\
\text { forma constante el } \\
\text { estado de situación } \\
\text { problema-actores-acciones. }\end{array}$ & $\begin{array}{l}\text { Preguntar/preguntar-se con y desde } \\
\text { actores y su carácter auto referencial. }\end{array}$ \\
\hline Programación & $\begin{array}{l}\text { Diseñar la lógica } \\
\text { de consistencia } \\
\text { acciones-actores-problema. }\end{array}$ & $\begin{array}{l}\text { Articular capacidad de respuesta con } \\
\text { el cuidado de articular las dimensiones } \\
\text { normativas y estratégica y de no } \\
\text { redimensionar el papel de la estrategia. }\end{array}$ \\
\hline organización & $\begin{array}{l}\text { Insuflar dinámica de } \\
\text { funcionamiento a la } \\
\text { capacidad de ejecución de } \\
\text { acciones-actores-problema. }\end{array}$ & $\begin{array}{l}\text { Coordinar arreglos organizativos, } \\
\text { mecanismos de comunicación y } \\
\text { espacios conjuntos de actuación para el } \\
\text { acceso a recursos y al funcionamiento } \\
\text { efectivo del apoyo logístico requerido. }\end{array}$ \\
\hline Evaluación & $\begin{array}{l}\text { Proveer evidencias en forma } \\
\text { constante del sentido de } \\
\text { la actuación problema- } \\
\text { actores-acciones (antes, } \\
\text { durante y después de la } \\
\text { toma de decisiones). }\end{array}$ & $\begin{array}{l}\text { Valorar el sentido, detectando cambios } \\
\text { o variaciones en los actores y su } \\
\text { actuación y derivando experiencias de } \\
\text { aprendizaje (estrategias preventivas } \\
\text { o de fortalecimiento y medidas } \\
\text { correctivas). }\end{array}$ \\
\hline
\end{tabular}

Fuente: elaboración propia con base en Pichardo, 2021.

En términos más específicos, y ampliando en alguna medida las conceptualizaciones precedentes, es posible señalar que, desde el modelo PipoE, la promoción es la base consustancial para ampliar, fortalecer, mejorar y lograr la mayor cantidad posible de adhesiones a favor, con predominio de confianza y colaboración; transformar la indecisión o indiferencia y el rechazo en contra (sobre la base de relaciones de conflicto y contraposición) o, al menos, neutralizar la interferencia. Un instrumento de apoyo para tal fin es el mapa de actores (partes interesadas y también partes desinteresadas) de constante y permanente actualización, y no únicamente como una especie de "fotografía" inicial. Su fin último es contribuir a consolidar una base social de apoyo de cara a re-pensando a la planificación como método de trabajo, desde una función prácticamente 
ausente en la planificación convencional, salvo estandartes publicitarios y otros signos externos propios del marketing comercial.

Con respecto a la indagación, se trata de un concepto que privilegiamos su uso para dar cuenta de una acción dinámica e integradora que va más allá del diagnóstico inicial, en tanto etapa previa a la planificación. Sin ánimos de caer en simplificaciones le conceptualizamos desde su significado esencial, es decir, intentar averiguar con preguntas, en un contexto en que, desde re-pensando la planificación como método de trabajo, interesa más saber hacer preguntas que obtener respuestas sin hacer preguntas. Sin perder de vista que una misma pregunta puede tener varias respuestas y una misma respuesta puede surgir a partir de diferentes preguntas. El instrumento sine qua non al servicio de la indagación es el estado o balance de situación. Su fin esencial es la valoración constante y en forma continua de las circunstancias en que se inscribe la planificación, tanto de nuevas situaciones que van aflorando como de otras que pueden seguir un curso distinto o diferente al inicialmente previsto. Y, de manera particular, contribuir a develar cuáles son las limitaciones para solventar, esto es, restricciones frente a la actuación, los problemas focales, vale decir, cuestiones que ameritan atención y los obstáculos a remover, entendidos estos últimos como inconvenientes o impedimentos con dificultad para ser removidos.

Por su lado, la programación, en tanto acción y efecto de programar, en ese sentido si se quiere ocupa el lugar de la planificación como usualmente se le tiende a identificar, pero con el cuidado de no convertir su práctica en acepciones convencionales, es decir, sin direccionalidad y sin criterio de viabilidad. El principal instrumento a su servicio es el Plan de Acción, en tanto favorece la delimitación de objetivos, el establecimiento de acciones, la definición de tiempos y la identificación de recursos. En ese sentido, es muy importante no perder de vista lo ya dicho sobre sus dimensiones interactuantes: la dimensión normativa, que se formaliza o concreta en un documento o guía de trabajo, y la dimensión estratégica, que forma parte integral de la evaluación en forma sistemática, permanente, constante y continua (y cualquier otro adjetivo que se le pueda endilgar para dejar claro que se trata de un proceso y no solamente de una etapa ex post o un balance intermedio).

En cuanto a la organización, desde el punto de vista semántico implica la acción y efecto de organizar y organizar-se. De manera que conlleva coordinar y procurar los medios adecuados para alcanzar los fines. Justamente, los dos conceptos básicos que deslindamos en el interior de su contenido: la coordinación y la administración. Al respecto, es útil subrayar que la clave de los nuevos tiempos es el trabajo colaborativo, que viene de colaboración e implica concurrir, trabajar activamente con otras personas, a diferencia de la simple cooperación. Es ahí donde cobra relevancia un concepto que hemos venido utilizando, promoviendo y divulgando:Aprendiencia, 'estar-en-proceso-de-aprender' mediante procesos de autoorganización colectiva e individual (Pichardo, 2017). 
Por último, aunque no en último lugar, la evaluación. Su etimología implica "estimar, calcular, apreciar el valor de algo". Desde re-pensando a la planificación como método de trabajo, esta función es el centro del accionar, como se representa en la rueda de timón o comando de la embarcación. Su papel principal es entroncar con evidencias, en particular de cara a la valoración de cambios o variaciones en la situación inicial, desde la perspectiva de aproximación a la situación deseada, en busca de obviar a la situación futura. Al respecto, es menester recalcar la necesidad de superar la tradición de considerar a la evaluación como una etapa final del proceso de planificación. Por el contrario, es necesario distinguir y practicarla en tres (3) momentos interrelacionados: evaluación ex ante, evaluación concurrente y evaluación ex post, esto es, antes, durante y después de la ejecución. Adquiriendo especificidad según el momento que se trate. De modo que a la evaluación ex ante le corresponde, en lo fundamental, identificar la viabilidad de posibles cursos de acción. Mientras que a la evaluación concurrente le compete la valoración de la ejecución y sus alcances. Y, por su lado, la evaluación ex post tendría que situarse en la valoración de la creación de condiciones para la sostenibilidad y replicabilidad a escala.

\section{Conclusiones}

A partir de este breve repaso desde re-pensando a la planificación como método de trabajo, y su fundamentación a partir de la categoría situación, la edificación del mapa situacional y el Modelo PipoE, es posible concluir que, con seguridad, esta forma de actuación forma parte integral de diversas prácticas profesionales, aunque no necesariamente sistematizadas y elaboradas bajo la forma del modelo presentado que, como tal, se trata de un ideal-tipo al estilo weberiano y, por tanto, su aplicación puede responder a diversidad de variantes situacionales.

Finalmente, agregaremos que la propuesta de re-pensando a la planificación como método de trabajo coloca a la evaluación en el centro, como ya hemos indicado. Se trata de la función que activa a las demás, al tiempo que se activa a sí misma, en confrontación permanente con la diversidad de situaciones, a diferencia o en oposición de las versiones convencionales de la planificación, cuyo "norte" es la definición de la norma. Aunque parezca un juego de palabras superpuestas, esto último significa que:

- La promoción se nutre de indagación, está sujeta a programación, supone organización y la evaluación es una constante para valorar pertinencia y efectividad.

- La indagación implica promoción, está sujeta a programación, supone organización y la evaluación es una constante para valorar alcances, resultados y aplicabilidad. 
- La programación es una derivación inicial de la indagación, implica promoción, supone organización y la evaluación es una constante para valorar calidad del diseño y efectividad de los resultados.

- La organización se deslinda de la programación, implica promoción, se nutre de indagación y la evaluación es una constante para valorar adecuación, efectividad y capacidad de adaptación ante situaciones no previstas o cursos de acción inesperados o inicialmente poco predecibles.

- La evaluación se deslinda de la indagación, implica promoción, está sujeta a programación, supone organización y, aunque parezca tautológico, la evaluación es una constante para valorar su capacidad de re/alimentar las demás funciones y a sí misma.

\section{Figura 3}

Interrelación entre las funciones esenciales del Modelo PipoE

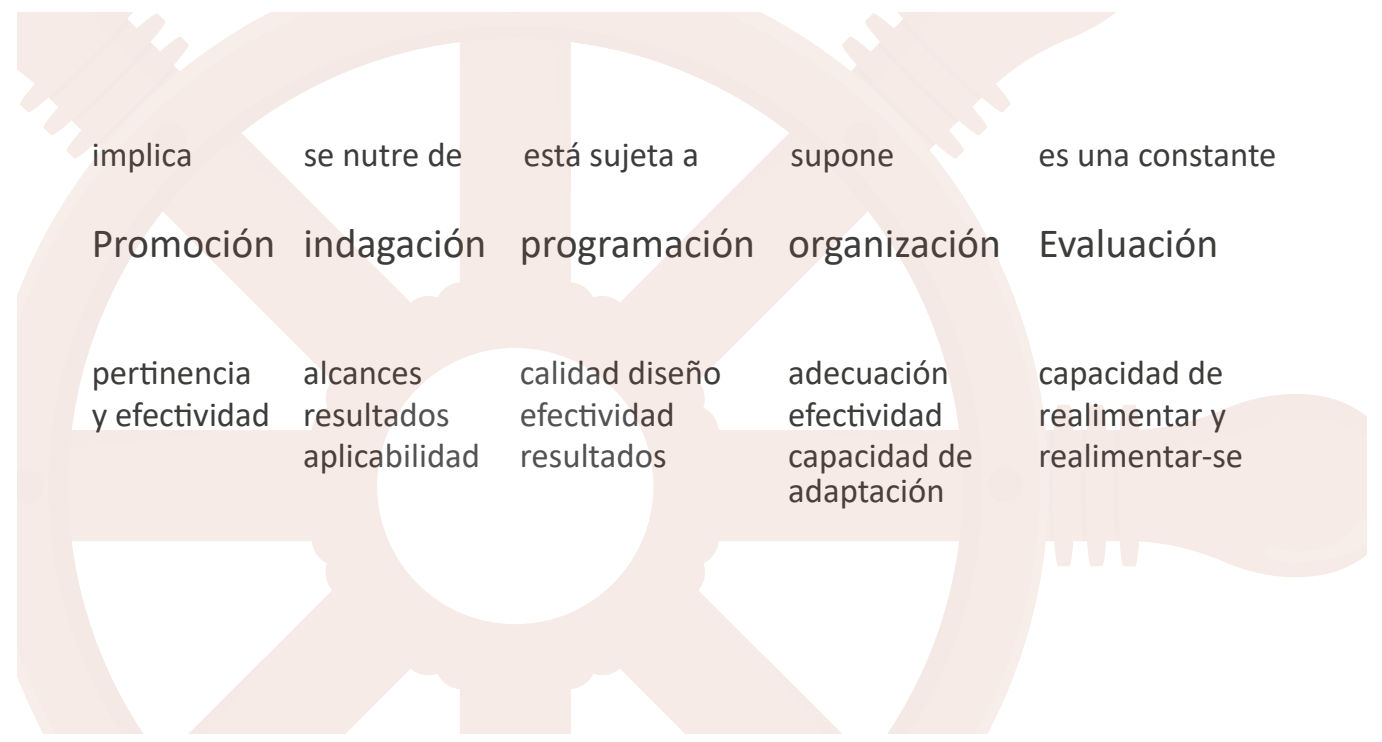

Fuente: elaboración propia con base en Pichardo, 2021, p. 164 


\section{Referencias bibliográficas}

Matus, C. (noviembre de 1981). Planificación en situaciones de poder compartido [Ponencia]. Seminario Estrategias y Paradigmas para el Desarrollo. Centro de Estudios del Desarrollo (Cendes) de la Universidad Central de Venezuela (UCV) y Programa de Naciones Unidas para el Desarrollo (PNUD).

Matus, C. (1977). Planificación de Situaciones. Libros Alfar.

Matus, C. (1972). Estrategia y Plan. Textos del Instituto Latinoamericano de Planificación Económica y Social (Ilpes). Editora Universitaria.

Pichardo, A. (2021). De cómo hacer Planificación Situacional aprendiendo. Editorial Brujas.

Pichardo, A. (2017). Ábreme la puerta. Casa de Aprendiencia de la Política Social y otras políticas. Editora Búho.

\section{Dirección de correspondencia:}

Arlette Pichardo Muñiz

Contacto: arlettepichardo@gmail.com

(CC) (2) Esta obra se encuentra bajo una Licencia de Creative Commons 